\title{
The sound of avalanches: from a global to a local perspective.
}

\author{
Florine Dubourg ${ }^{1, \dagger}$, Sébastien Lherminier ${ }^{1, \dagger}$, Ramon Planet $^{2}$, Kenny Rapina ${ }^{1}$, Félix Bunel ${ }^{1}$, Loïc Vanel ${ }^{1}$, and \\ Osvanny Ramos ${ }^{1, \star}$ \\ ${ }^{1}$ Univ Lyon, Université Claude Bernard Lyon 1, CNRS, Institut Lumière Matière, F-69622, Villeurbanne, France \\ ${ }^{2}$ Departament de Física de la Matèria Condensada, Universitat de Barcelona, Martí i Franquès 1, E-08028 Barcelona, Spain
}

\begin{abstract}
We present an experimental study aiming to understand the local origin of the acoustic bursts recorded globally during a two-dimensional shear experiment of a granular medium. In order to explain the power-law distribution of energy of these events (also called avalanches), the analysis focuses on their acoustic frequencies. Experiments with single grains have also been performed, trying to separate the contributions related to collision between neighbours from shear movements between grains. Preliminary results indicate that both frictional sliding and collision-like mechanisms are involved in the origin of the acoustic bursts. The evolution of the structure of the medium around a large acoustic burst is analysed both with high resolution and high-speed imaging. The energy of the acoustic bursts seems related to single and well-localised events with very large variations in energy. However, these local reorganisation processes may eventually bring the global relaxation of the system, which is also captured by a force drop in the resistance to shear.
\end{abstract}

\section{Introduction}

Shearing compressed granular materials has often been used as a simplified model of a tectonic fault. In most real [1-3] or numerical experiments [4-7], two parallel plates (mimicking tectonic ones) compress the granular system. As the plates move laterally in relation to each other and at very low speeds, shear stresses build up on the packed grains, and eventually they are liberated through sudden events associated to the reorganisation of the pack. These events -often called avalanches- may be detected from sudden jumps in the global shear force experienced by the plates $[2,8]$, from acoustics bursts $[1,9]$ or even from direct visualisation in the case of photoelastic grains [2, 3]. One weakness of most shear experiments, that limits the comparison with real earthquakes, is their lack of statistics. However, we have recently built a shear experiment that, thanks to the implementation of periodic boundary conditions, is able to reach significant statistics of more than $10^{6}$ events [9]. The energies of our "avalanches", measured as acoustic bursts, follow a five-decades power law with an exponent of 1.72 , which is close to the one of the Gutenberg-Richter law, equal to $5 / 3=1.66$ [10]. Other earthquake laws have also been reproduced. However, many new questions have arisen related to the local origin of the recorded acoustic events.

Are those events the result of "snow-ball" or "domino" effects where many different parts of the system are reached by the event and become part of it? In this case the term avalanche is appropriate. Or instead are the huge

\footnotetext{
$\dagger$ These authors contributed equally to this work

^e-mail: osvanny.ramos@univ-lyon1.fr
}

variations in energy only associated to single and welllocalised events? Like, for example, the breaking of single structures with a large distribution of sizes [11]. Another question focuses on the local mechanism of the globally recorded sound: is it a signature of a collision between grains, or does the frictional sliding between the grains' rough surfaces provoke it? At intermediate scales between the grains and the whole system, the force network plays an essential role in the stability of the granular packing [12-14]. What is the relation between their dynamics and the recorded acoustic bursts? In order to tackle these questions, we have built a simplified version of our experiment consisting of a classical shear cell. The experiments will focus on the details of one single avalanche.

\section{Main Setup}

The experimental setup consists of a monolayer of disks confined into a rectangular shear cell (figure 1) which has an initial size of $172 \times 105 \mathrm{~mm}^{2}(W \times H)$. In order to avoid $3 \mathrm{D}$ effects, the cell is confined between two acrylic plates separated by $4.2 \mathrm{~mm}$. The surrounding walls are built in the same material as the grains. The bottom wall is fixed, while the upper and lateral ones are mobile, allowing the shearing of the granular medium. The upper plate is free to move vertically, compressing the medium. A dead load of $120 \mathrm{~N}$ is applied during the whole experiment. This plate is also connected to a force sensor (Interface $S M L-50)$, which is pulled at a constant speed of $100 \mu \mathrm{m} / \mathrm{s}$. We use cylindrical grains of $4 \mathrm{~mm}$ thickness and two different diameters 6.4 and $7 \mathrm{~mm}$ (in equal proportion). These discs (the same as the ones used in [9]) 


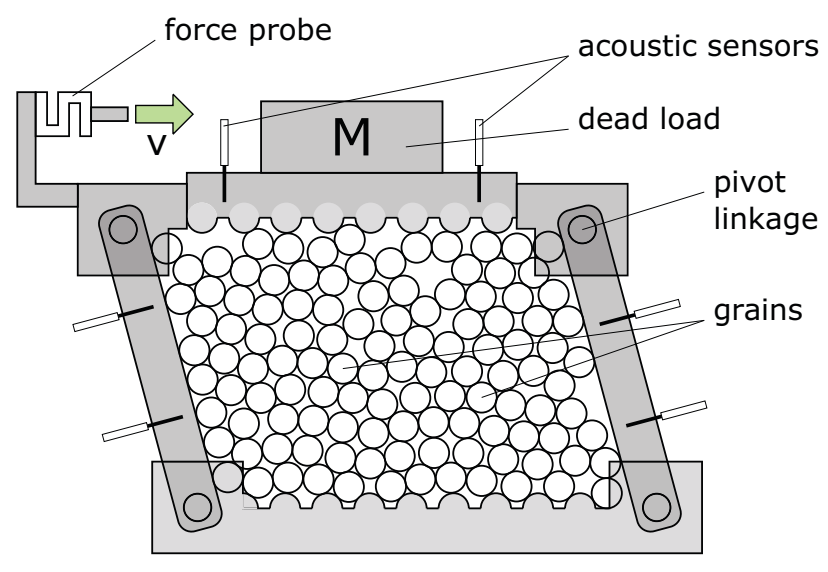

Figure 1. (colour online) Schema of the main experimental setup.

are made of Durus White 430 and have been generated in a Objet30 3D-printer. Profile measurements of the surface roughness of the printed grains give a peak-to-peak maximum value of $25 \mu \mathrm{m}$ on the curved side of the cylinders. The zero-frequency Young modulus of Durus material is $E \simeq 100 \mathrm{MPa}$. The cell contains 384 grains placed in a disordered configuration, reached thanks to half grains of diameter $d=6.4 \mathrm{~mm}$ printed in the top and bottom plates with a specific gap of $\sqrt{2} d$ [15]. The translucent and photoelastic character of the grains allows the visualisation of the stress inside the discs when placing the experimental setup between two circular polarizers. We take images of the granular packing with a Nikon D750 high resolution camera $(6016 \times 4016$ pixels $)$ at $1 \mathrm{fps}$ during the whole experiment and with a Phantom V711 fast camera $(512 \times 256$ pixels), at 49,000 fps, triggered around one large acoustic event.

Acoustic emissions (AE) are recorded using 6 piezoelectric pinducers (VP-1.5 from CTS Valpey Corp.) inserted in adjusted holes in the upper and lateral walls. Both the force and the acoustics are recorded with a NI-USB6366 card at $100 \mathrm{kHz}$ during the whole shear movement.

\section{Results}

\subsection{Acoustic analysis}

A typical acoustic signal is shown in the figure $2 \mathrm{a}$. The $\mathrm{AE}$ are analysed through a time-frequency method: we computed the spectrogram of the acoustic signal of each sensor. A spectrogram (figure $2 b$ ) is obtained by a sliding Fourier transform in a time window of $1.28 \mathrm{~ms}$. It represents the energy of the signal for a set of frequencies linearly distributed between 0 and the Shannon frequency ( 50 $\mathrm{kHz}$ ). By thresholding the logarithm of the intensity of the spectrogram (colour code), and finding the local maxima, it is possible to obtain the main frequencies emitted during the avalanches. The histogram of the frequencies emitted during five different shear experiments is presented in the figure $2 \mathrm{c}$. We observe that a few frequencies are more represented, respectively around $2900 \mathrm{~Hz}, 4800 \mathrm{~Hz}$ et 8600 $\mathrm{Hz}$.
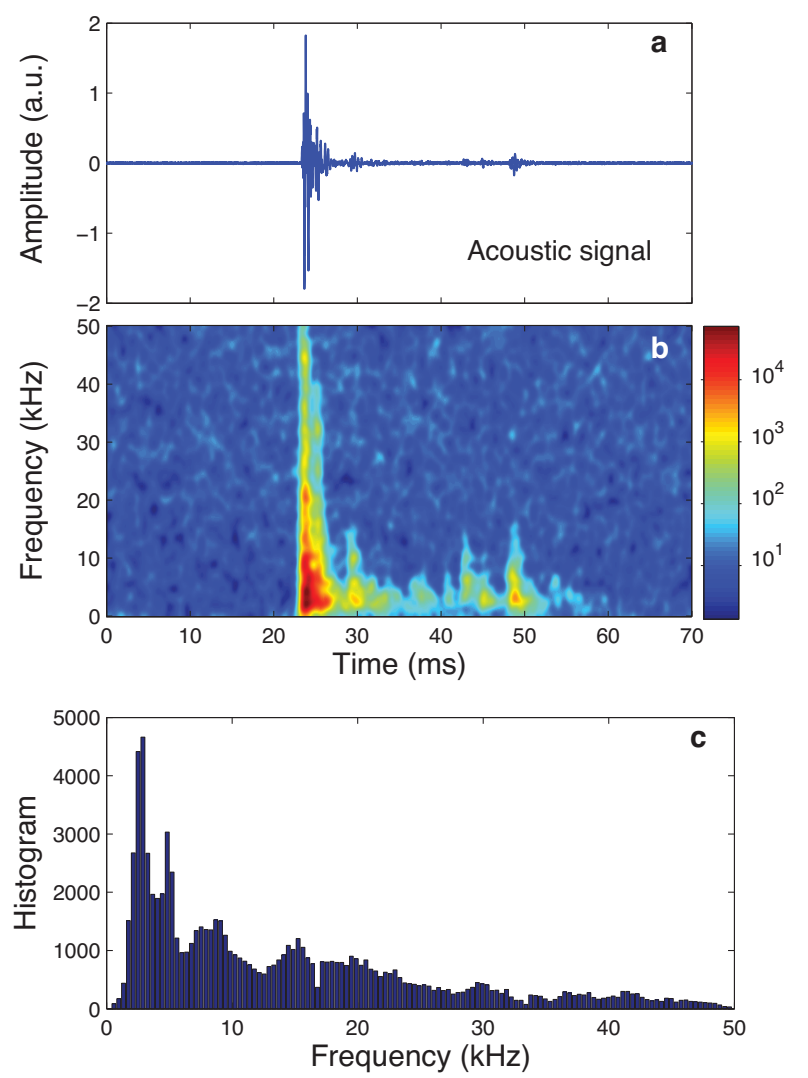

Figure 2. (colour online) a. Typical acoustic signal recorded during a shearing experiment, on a $70 \mathrm{~ms}$ time window. b. The associated spectrogram on 256 frequencies. c. Histogram of detected energy peaks on the spectrograms for a set of five different shearing experiments.

\subsection{Image analysis}

The analysis of high-resolution images (figure 3a) allows the precise detection of the position of the grains and, after a calibration of the photoelasticity, the force experienced by each contact point of every grain inside the packing. The precision of these force measurements is low. However, good enough to detect the grains involved in force chains [12-14] (figure 3). A simple analysis of the intensity variations along a ring excluding the central part of the grain is able to detect the contacts holding a high force value. It is known that acoustic waves propagate preferentially through force chains $[14,15]$. Here we show also that the acoustic source of large acoustic bursts is localised along force chains.

By analysing the series of high-speed images we are able to determine the changes in the internal structure. Any change in the force applied at a grain contact will manifest itself by a change in the light intensity recorded by the camera. By examining the differences of intensity pixel by pixel during an avalanche, we are able to resolve the propagation of the reorganisation. On figure $3 b$, we represent all the detected changes of intensity for respectively a time of $0.25,1$ and 2 milliseconds since the beginning of the avalanche. We observe a good similarity between the pixels that experienced a change of value and the ones corresponding to force chains as detected on the 

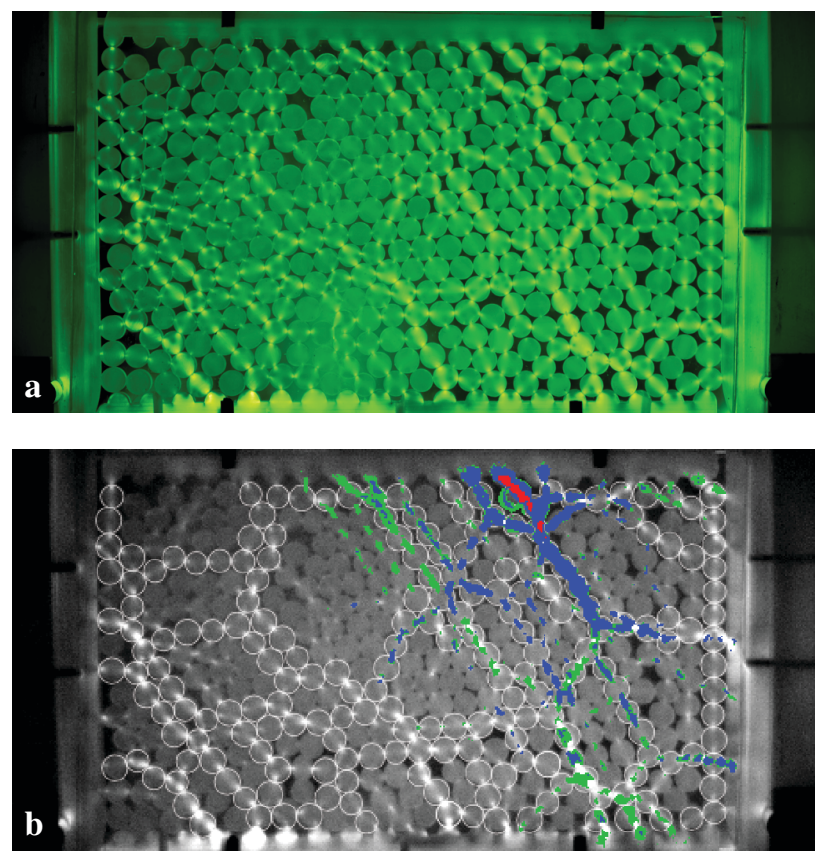

Figure 3. (colour online) a. High-resolution image used for force chains detection. A green filter has been used. b. Highspeed image of the cell with highlighting (white colour) of grains involved in force chains. Colors represent pixels whose intensity changed since the beginning of the avalanche, depending on the time elapsed: red is for $0.25 \mathrm{~ms}$ since the beginning of the avalanche, blue for $1 \mathrm{~ms}$, and green for $2 \mathrm{~ms}$.

high-resolution images. We also see that the avalanche begins locally, involving only a few grains belonging to one short force chain, and then the structure relaxes on a large scale during the next few milliseconds. This relaxation is captured also by a force drop in the resistance to shear. The same behaviour have been noticed in all the other analysed events.

\subsection{Collisions vs. Shearing}

In order to compare the $\mathrm{AE}$ produced by either collisions between grains or frictional sliding of their contacts, we designed two experimental setups that each reproduce one of the mechanisms. The first one (figure 4a) consists of one slider with controlled mass freely moving vertically in a guide and with a Durus cylinder glued at its bottom. When the slider is released from a certain height, it will accelerate and hit another grain placed on the floor. The $\mathrm{AE}$ is recorded thanks to a pinducer stuck to the side of the immobile grain.

The second setup (figure $4 \mathrm{~b}$ ) aims to reproduce the frictional sliding of the grains contacts. A free grain is confined between two pairs of grains, one pair glued to a fixed stand and the other freely moving vertically and supporting a dead load. Thanks to a hole drilled in the free grain, we use a wire to extract it quickly (by hand so without a control of the speed) from its nest. The upper pair of grains is stopped before touching the inferior ones. Again the $\mathrm{AE}$ is recorded by a pinducer stuck to one of the still grains.
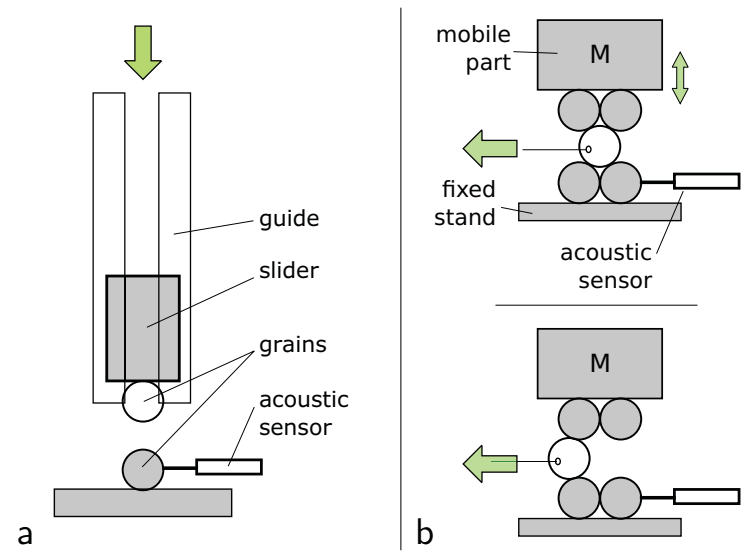

Figure 4. (colour online). Experimental setups used to reproduce the two acoustic emissions mechanisms, respectively the impacts (a) and the frictional sliding (b).

Acoustic bursts emitted by both mechanisms are analysed in the same way we did for the AE of the shearing experiments, with a time-frequency analysis. These spectrograms are represented on figure $5 \mathrm{a}-\mathrm{d}$. We note that the main frequencies of the $\mathrm{AE}$ are much better defined in this case, since the wave is much more confined in one or two grains. Again we are able to extract the frequencies emitted in all the experiments performed and plot their histograms on figure $5 \mathrm{e}-\mathrm{f}$. We observe different frequencies emitted by the two mechanisms, with a main peak at 2400 $\mathrm{Hz}$ for the impacts and one at $4800 \mathrm{~Hz}$ for the frictional sliding.

\section{Discussion \& Conclusion}

The first and second peaks appearing in the histogram of figure $2 \mathrm{c}$ seem to correspond to the main peaks obtained in the collision of grains (figure 5e) and the frictional sliding (figure 5f) respectively. This result indicates that both mechanisms, collisions and frictional sliding, play important roles at the origin of the acoustics events. As frictional forces are responsible for the stability of the system, a local sliding at the contact interface between two grains may be the starting point of the process. Large force values may be required for provoking this failure, which would be consistent with the localisation of the origin of large events along force chains. The sliding of the grain will suddenly change the local distribution of forces, which is equivalent to a collision.

This instability propagates in the system, particularly along force chains (figure $3 \mathrm{~b}$ ) like a "domino" effect, provoking a reorganisation of both the structure and stress in the granular medium. However it concerns just a few dozens of grains in the rare case of a very large event; thus it cannot explain the existence of a power-law distribution of acoustic energies as "snow-ball" effects [16, 17]. Instead, one (or more) of the local events described in the previous paragraph may dominate in terms of energy release, and it is the signature of this local release of energy that accounts for the energy distribution. 

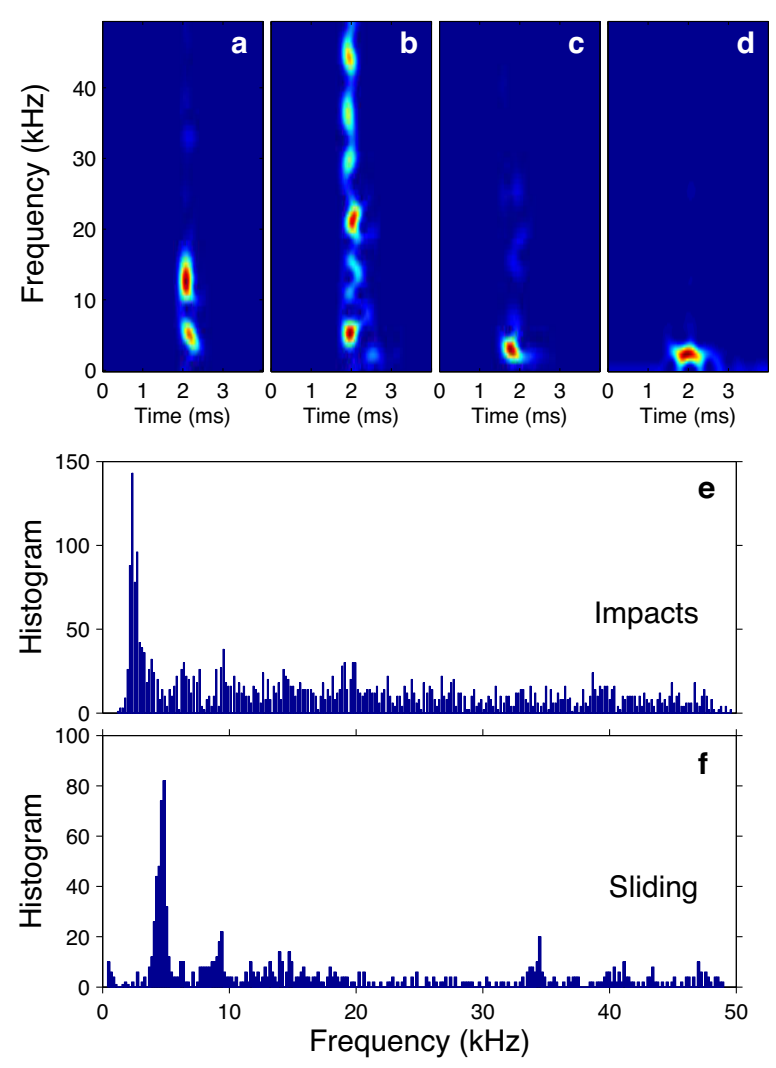

Figure 5. (colour online) a. Spectrogram of the AE produce by frictional sliding. b, c, d. Spectrograms of the AE produced by impacts of decreasing speeds (resp. 2.8, 0.3 and less than $0.1 \mathrm{~m} / \mathrm{s}$ ). e, f. Histograms of frequencies of spectrograms peaks recorded for a group of experiments of impacts and frictional sliding respectively.

In the case in which the main energy contribution comes from a collision-like effect, we could consider the phenomenon as a single and well-localised event. However, the frictional sliding is a much more complicated process $[18,19]$. Although the energy captured by the acoustic also comes from single and well-localised events, it is difficult to judge if there is (or not) a microscopical avalanche-like process of breaking of asperities at the origin of the local frictional failure.

In conclusion, our experimental results indicate that both frictional sliding and collision-like mechanisms are involved as the origin of the acoustic bursts recorded globally during the shear of our granular system. The wide distribution (power-law) found for the acoustic energies has to be understood in term of of single and well-localised events with very large variations in energy happening preferentially along force chains. Possibly, these local reorganisations may propagate, bringing a global relaxation of the system.

\section{Acknowledgements}

We thank G. Simon for technical support. We acknowledge financial support from the AXA Research Fund and the Chair-CNRS program.

\section{References}

[1] P.A. Johnson, H. Savage, M. Knuth, J. Gomberg, C. Marone, Nature 451, 57 (2008)

[2] K.E. Daniels, N.W. Hayman, Journal of Geophysical Research: Solid Earth 113 (2008)

[3] D.M. Walker, A. Tordesillas, M. Small, R.P. Behringer, K.T. Chi, Chaos: An Interdisciplinary Journal of Nonlinear Science 24, 013132 (2014)

[4] K. Mair, C. Marone, Journal of Geophysical Research: Solid Earth 104, 28899 (1999)

[5] O. Sotolongo-Costa, A. Posadas, Physical Review Letters 92, 048501 (2004)

[6] I. Bratberg, F. Radjai, A. Hansen, Phys. Rev. E 71, 011301 (2005)

[7] B. Ferdowsi, M. Griffa, R.A. Guyer, P.A. Johnson, C. Marone, J. Carmeliet, Geophysical Research Letters 42, 9750 (2015), 2015GL066096

[8] R. Planet, S. Lherminier, G. Simon, K. Måløy, L. Vanel, O. Ramos, Mimicking earthquakes with granular media, in 22ème Congrès Français de Mécanique (2015)

[9] S. Lherminier, R. Planet, G. Simon, L. Vanel, O. Ramos, Revista Cubana de Física 33, 55 (2016)

[10] T.C. Hanks, H. Kanamori, Journal of Geophysical Research B 84, 2348 (1979)

[11] M. Stojanova, S. Santucci, L. Vanel, O. Ramos, Physical Review Letters 112, 115502 (2014)

[12] B. Miller, C. O'Hern, R. Behringer, Physical Review Letters 77, 3110 (1996)

[13] T.S. Majmudar, R.P. Behringer, Nature 435, 1079 (2005)

[14] E.T. Owens, K.E. Daniels, Europhysics Letters 94, 54005 (2011)

[15] S. Lherminier, R. Planet, G. Simon, L. Vanel, O. Ramos, Physical Review Letters 113, 098001 (2014)

[16] P. Bak, C. Tang, K. Wiesenfeld, Physical Review Letters 59, 381 (1987)

[17] O. Ramos, E. Altshuler, K. Måløy, Physical Review Letters 102, 078701 (2009)

[18] S.M. Rubinstein, G. Cohen, J. Fineberg, Physical Review Letters 98, 226103 (2007)

[19] O. Ben-David, G. Cohen, J. Fineberg, Science 330, 211 (2010) 